\title{
Advancement of Principal Component Judgment for the Classification and Prediction of Alzheimer's Disease
}

\author{
M.S.Roobini, M.Lakshmi
}

\begin{abstract}
Alzheimer is a dynamic issue of dementia, which deals with mind issue that assaults synapses, cerebrum cells, and nerves, memory, and practices and afterward finally causing dementia on old individuals. In spite of its significance, there is at present no remedy for it. In any case, there are drugs accessible on remedy that can help defer the advancement of the condition. Principal Component Analysis is an incredible system to recognize the examples of vast informational indexes, offers an in vogue factual strategy to dissect multivariate information by building a brief information portrayal utilizing the predominant Eigen vectors of the information covariance lattice. Along these lines, early conclusion of $A D$ is basic for patient consideration and pertinent examines. In this paper, we have assessed a calculation utilizing Principal Component Analysis for its application in information investigation. In the exploration field, it is exceptionally hard to comprehend the expansive measure of information and is very tedious as well. In this way, so as to maintain a strategic distance from wastage of time and for the simplicity in understanding we have examined a PCA calculation that can diminish the gigantic component of the information. Principal Component Analysis (PCA) has been utilized in this paper to locate the base number of credits to improve the classifiers for quicker execution, cost-adequacy and precision. The strategy for PCA is utilized to pack the greatest measure of data into initial two sections of the changed lattice known as the vital parts by ignoring alternate vectors that conveys the immaterial data or repetitive information. Utilizing PCA we expect to locate the important highlights of the informational indexes. This paper proposes a system for forecast of Alzheimer sickness by discovering the most critical highlights important to Alzheimer Disease and furthermore different therapeutic picture application-based PCA results are displayed to demonstrate its productivity.
\end{abstract}

Keywords- Principal component Analysis, Alzheimer, Feature Extraction, Feature Selection, Support Vector Machine, and Linear Discriminate Analysis.

\section{INTRODUCTION}

The noteworthy headways in organic sciences have made amazing improvements in numerous fields including medicinal picture the board. This kind of restorative pictures is the fundamental piece of specialists and radiologist to analyze tolerant case. Advanced Imaging and Communications in Medicine has been an all-inclusive standard for verified correspondence of therapeutic substance over systems. This Principal Component Analysis is an incredible system to distinguish the examples of expansive informational collections, offers an in vogue factual technique to break down multivariate information by

Revised Manuscript Received on July 10, 2019.

M.S.Roobini, Computer Science and Engineerring,Sathyabama Institute of Science and Technology, Chennai, T.N, India.

Dr.M.Lakshmi,Computer Science and Engineering,Sri Krishna College of Technology, Coimbatore. T.N, India. developing a brief information portrayal utilizing the overwhelming Eigen vectors of the information covariance network. Principal Component Analysis is frequently utilized to diminish the elements of information before applying progressively modern information investigation techniques, for example, non-straight characterization calculations or free part examination. It includes the count of the EVD of an information covariance network or SVD of an information grid, more often than not after mean focusing the information for each element. It is the least difficult of the genuine Eigen vector-based multivariate examination. Regularly, its task can be thought of as uncovering the inward structure of the information in a manner which best clarifies the difference in the information.

This investigation of PCA is a scientific technique which utilizes complex numerical standards to change various related factors into fewer factors called key parts. In PCA, the data contained in a lot of information is put away with decreased measurements dependent on a subspace created with an arrangement of symmetrical tomahawks. The diminished measurements computational substance is chosen with the goal that the huge information qualities are related to little data misfortune. Such a decrease is favorable position in a few fields concerning picture pressure, information portrayal, and so on. It can likewise be generally utilized for highlight extraction, picture combination, picture pressure, picture division, picture enrolment, de-noising, and so on.

Neuro-imaging ponders normally gather information from various modalities.PCA is a standard procedure for imagining high dimensional information and for information pre-preparing. PCA diminishes the dimensionality (the quantity of factors) of an informational index by keeping up however much change as could be expected. Utilizing PCA, we can recognize the two-dimensional plane that ideally portrays the most astounding information difference.

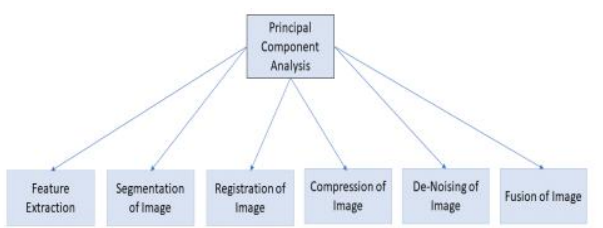

Fig 1: Usage of Principal Component Analysis in Medical Imaging

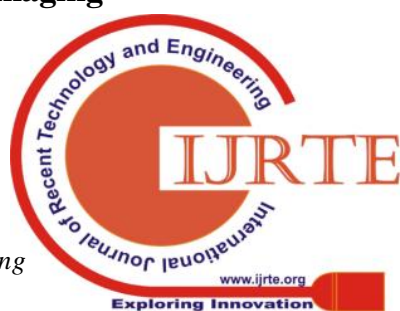




\section{ADVANCEMENT OF PRINCIPAL COMPONENT JUDGMENT FOR THE CLASSIFICATION AND PREDICTION OF ALZHEIMER'S DISEASE}

Connections between these diverse Neuro-imaging estimations and clinical or intellectual test outcomes. This paper shows that ordinary PCA as an exploratory information preparing venture for dimensionality decrease in characterization studies may not be ideal. A substitute strategy for requesting PCA eigenvectors is proposed which has better biased qualities. This strategy is connected to a FA informational collection from schizophrenia subjects and sound controls, trailed by further investigation to recognize white issue tracts for segregating these two gatherings. In the clinical condition there is a critical development in the measure of information produced and gathered. Innovation has assumed a noteworthy job in the development of our capacity to break down and comprehend the information to analyze illnesses and for early treatment. For such basic leadership it is fundamental to mine the clinical information.

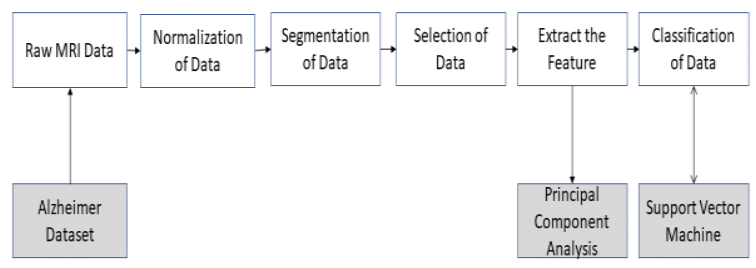

Fig 2: Architecture of the Proposed System

Different Data mining strategies are accessible to get new examples from the dataset by joining various information sources Clinical information are valuable to foresee different maladies, give appropriate treatment to fix illness, decrease death rate and help the legislature to detail national projects to improve personal satisfaction and keep away from preventable passing. Our investigation talks about use of Principal Component Analysis (PCA) to locate the applicable highlights of the informational indexes and troupe structure for maladygrouping and forecast with three layered methodology. Indeed, even inside one methodology of MRI.

The PCA is a notable information decrease instrument in the scholarly world for more than 100 years. PCA makes a straight symmetrical change of associated information in one casing (organizes framework) to uncorrelated information in another casing. The enormous dimensional information can be changed and approximated with a couple of measurements. PCA finds the bearings of greatest difference in high-dimensional information and tasks it onto a littler dimensional subspace while holding a large portion of the first data. On the off chance that the information is loud, PCA lessens clamor verifiably while anticipating information along the key segments. In this paper, we investigate a versatile cross breed way to deal with demonstrate that PCA can be utilized for information decrease as well as for relapse calculation improvement.

\section{PCA algorithm:}

PCA is an excellent method for removing high dimensional dataset. Actually, this can be performed by taking care of Eigen esteem issue, or utilizing iterative calculations to assess the central parts. It is considered as a symmetrical change in which the information will be depicted where used to change a lot of corresponded factors into a lot of uncorrelated factors. The new dataset values are called important segments. In PCA, the biggest conceivable change can be found in the principal part. Following parts having the most noteworthy difference conceivable alludes to the segments being symmetrical to the past segments. PCA ends up free if the dataset is dispersed together, and furthermore touchy to scaling of unique factors.

\section{PCA applications in medical care using image processing:}

Foremost parts examination is a technique that figures the together symmetrical headings of most extreme difference in a gathering of $n$-dimensional information. These $d$ headings structure a symmetrical premise that degrees the information space.

\section{RESULTS \& DISCUSSIONS}

\section{Calculation: PCA for Compression:}

Stage 1: Get the element section vector lattice from the given picture information.

Stage 2: Get the covariance network A.

Step3: Use trademark condition $(\lambda n-E B)=0$ to get the Eigen values. These Eigen values structure the network Ez.

Stage 4: Considering the Eigen values to compute the eigenvector network.

Stage 5: Obtain the change WT by considering the eigenvectors as their sections.

Stage 6: Obtain the highlights vector framework by $\mathrm{Cd}=$ CAWT.

Stage 7: For pressure of a picture, the dimensionality of the new component vector has diminished by setting little Eigen values 1 to zeros.

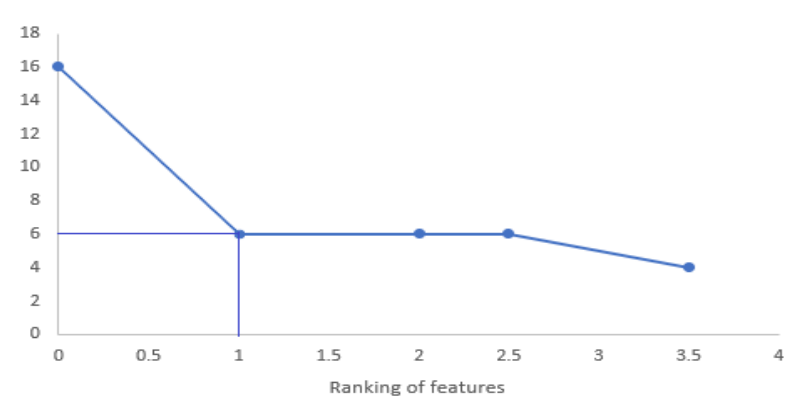

Fig 3 : Values of the Selected Feature for AD Classification

Compression of Image in Medical Care:

It assumes a fundamental job in restorative applications. The primary point of picture pressure is to expel the excess from picture to permit a similar picture remaking at the collector end. There are primarily two sorts of picture pressure strategies, lossy and lossless. Computerized or video pictures are compacted by lossy pressure strategies. Then again, as each piece of data is significant, in therapeutic applications like X-beam, the pictures are packed by lossless pressure strategies where CT and MRI imaging systems results in a lot of information which

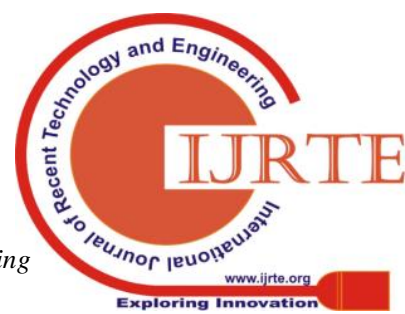


requests a picture pressure technique. The PCA calculation for information change and information pressure is abridged as pursues. Speak to the picture information by a lot of $\mathrm{m}$ vectors, $\mathrm{A}=[\mathrm{a} 1, \mathrm{a} 2 \ldots$ ai, $\mathrm{aM}]$, where ai speaks to $\mathrm{n}$ highlights. Consequently, the dataset A speaks to the component section vector 1 . It is required to register the information combination network an and extricate the Eigen esteems and Eigen vectors. All the element section vectors are gathered in a grid $\mathrm{A}$.

\begin{tabular}{llll}
\hline Techmiques & Paper Aufhor/Year & Disease & Accuracy \\
\hline Naive Bayes & lyer \& sheen $/ 2015$ & AD & $79.56 \%$ \\
Supervised Laaming & Catherine/2013 & AD & $75 \%$ \\
Naive Bayes & Stephen & AD & $77.89 \%$
\end{tabular}

Table 1:Some of the Machine Learning Techniques used for automatic diagnosis of $\mathrm{AD}$

The prescient estimation of 18F-fluorodeoxyglucose (FDG) PET for change from mellow psychological weakness (MCI) to Alzheimer's dementia (AD) is at present under discussion. We utilized an essential segment examination (PCA) to recognize a metabolic $\mathrm{AD}$ transformation related example (ADCRP) and explored the prognostic estimation of the subsequent example articulation score (PESCalculation to dissect auxiliary attractive reverberation imaging information; utilizing this framework, we can anticipate transformation of mellow intellectual disability (MCI) - to-Alzheimer's illness (AD) at somewhere in the range of one and three years before clinical analysis. The CAD framework was created in four phases. To start with, we utilized a Voxel-based morphometry method to research worldwide and nearby dim issue (GM) decay in an $\mathrm{AD}$ bunch contrasted and sound control (HCs). Locales with critical GM volume decrease were portioned as volumes of intrigue (VOIs).

\begin{tabular}{|c|c|c|}
\hline Selection of Features & $\begin{array}{c}\text { No of } \\
\text { Features }\end{array}$ & Accuracy \\
\hline Positive Proposed Methodology & 2085 & $96.74 \%$ \\
\hline Negative & 37189 & $81.34 \%$ \\
\hline
\end{tabular}

Table 2: Results of AD

The Voxel values were then separated into an element vector. Third, at the highlight determination organize, all highlights were positioned by their particular t-test scores and a hereditary calculation intended to locate the ideal element subset. The Fisher standard was utilized as a major aspect of the target work in the hereditary calculation. At long last, the arrangement was completed utilizing a help vector machine (SVM) with 10-overlay cross approval. We assessed the proposed programmed CAD framework by applying it to standard qualities from the Alzheimer's disease Neuro-imaging Initiative (ADNI) dataset (160 AD, $162 \mathrm{HC}, 65 \mathrm{sMCI}$ and $71 \mathrm{pMCI}$ subjects). The exploratory outcomes showed that the proposed framework is prepared to do recognizing sMCI and pMCI patients, and would be proper for commonsense use in a clinical setting.Alzheimer's illness (AD) is a type of dynamic irreversible dementia that happens most every now and again in more established grown-ups. Advertisement step by step destroys the locales of the cerebrum that are in charge of memory, considering, learning, and other psychological capacities [13]. It has been evaluated that the quantity of patients who experience the ill effects of $\mathrm{AD}$ will twofold in the following two decades, and will contact 13.8 million individuals by 2050 [14]. In America, AD is one of the main 10 reasons for death that can'tbe restored or anticipated [13]. Early location may elucidate the components fundamental $\mathrm{AD}$ and to improve the personal satisfaction for $\mathrm{AD}$ patients [13].

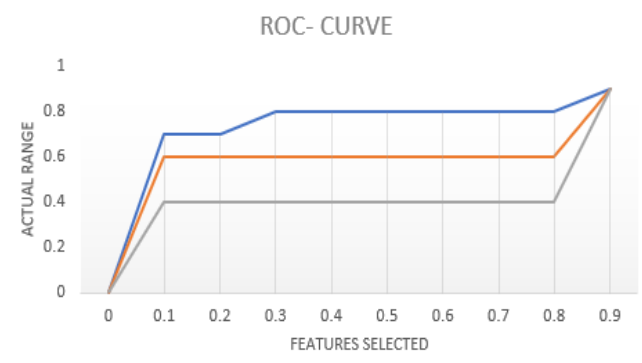

Fig 4: ROC Curve of the method used

Dementia is a syndrome due to disease of the brain, usually chronic, characterized by a progressive, global deterioration in intellect including memory, learning, orientation, language, comprehension and judgment. Alzheimer's disease (AD) is a one of the most important examples of dementia which mostly affects people over 65 years old and whose incidence rate grows exponentially with age, almost doubling in every 5 years. Still, apart from a few exceptions, the factors that trigger the onset of $\mathrm{AD}$ remain unknown. It is a progressive disease, this means that it worsens over time.

\begin{tabular}{|lrclc|}
\hline Diagno is & $\begin{array}{r}\text { No of } \\
\text { Samples }\end{array}$ & \multicolumn{1}{c}{ Age } & Gender(MaleFemale) & Range \\
\hline NC & 210 & $75 \pm 10$ & $102 / 120$ & $28.03 \pm 1.5$ \\
AD & 465 & $74 \pm 7$ & $230 / 185$ & $25 \pm 2.3$ \\
MCI & 180 & $72 \pm 8$ & $98 / 108$ & $22 \pm 20$ \\
\hline
\end{tabular}

Fig 5: Demographic table of Patients in AD Dataset

Structural and Functional Neuro-imaging allow studying of brain pathology at macro and micro molecular level like Magnetic Resonance Imaging (MRI), single photon emission computed tomography (SPECT), and Positron emission tomography (PET), table 1 shows different types of Neuro-imaging.

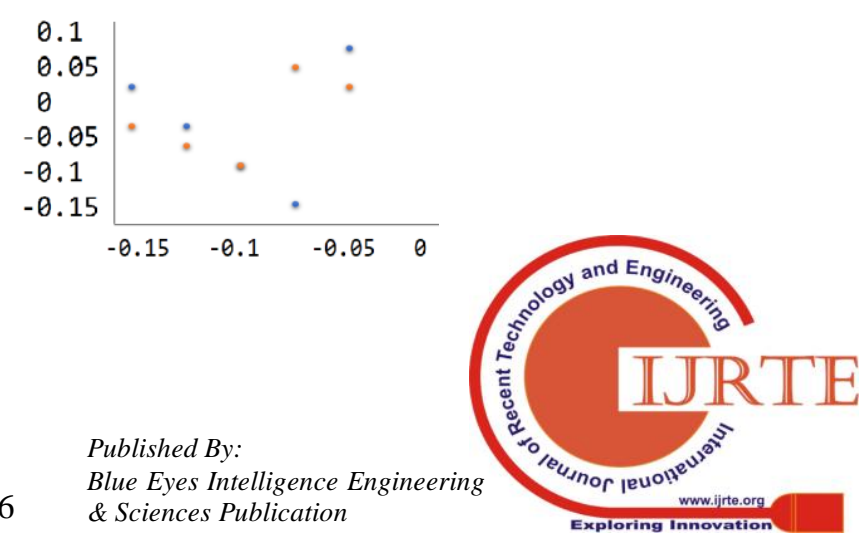




\section{ADVANCEMENT OF PRINCIPAL COMPONENT JUDGMENT FOR THE CLASSIFICATION AND PREDICTION OF ALZHEIMER'S DISEASE}

Fig 6: Scores of Principal Component Analysis

\section{Literature Survey:}

[1] Principal Component Analysis (PCA) has been utilized in this paper to locate the base number of ascribes to upgrade the classifiers for quicker execution, cost-adequacy and exactness. Utilizing PCA we expect to locate the important highlights of the informational indexes. A gathering order structure is utilized which comprises of three modules, in particular information procurement and pre-preparing, classifier preparing and Hierarchical Majority Voting (HMV) outfit model for illness grouping and prediction. Non-Communicable maladies are a worldwide weight which is expanding the death rate of individuals in rustic areas. Early identification through computerized framework lessens social insurance cost as screening and forecast are done at patient's entryway step. This examination additionally demonstrates that order and expectation of sicknesses utilizing HMV approach accomplishes most elevated exactness. This gives proof based mechanical methodology and furthermore fills in as a model for the up and coming national projects for the arrangement producers in the executives of NCDs.

[2] Here the PCA was performed by utilizing connection with therapeutic information. Factors relating to concoction trial of blood in particular lipoprotein, triglyceride, Apo protein A-1, Apo protein B, phospholipids, all out lipid, glucose and uric corrosive, are embraced to know the connection among them and enrollment of gathering variable. The outcomes showed that among these components' cholesterol, triglyceride, Apo protein B, low thickness lipoprotein, phospholipids, all out lipid and uric corrosive were recorded to be higher in IHD bunch contrasted with those of control gathering. High thickness lipoprotein and Apo protein A-1 were recorded to be lower in IHD gathering, though discovered higher in control gathering. Cholesterol was exceedingly corresponded with low thickness lipoprotein and tolerably related with all out lipid.

The initial four parts have clarified 60.67 percentage segment variability. In the instance of discovering connections between factors, it was seen that Cholesterol was profoundly related with low thickness lipoprotein and decently corresponded with absolute lipid. Against picture connections translated that the factors were moderate. To know the fluctuation between the parts, it was discovered that initial four segments represent precisely $60.67 \%$ of the absolute inconstancy. These four segments sufficiently depict the entire relationship grid. At long last it was clear to see that cholesterol; Apo protein B and low thickness lipoprotein had high esteem and has a place with the segments 1 The factors had high weight and had a place with part 4 were high thickness lipoprotein and glucose. The significant finding of this examination is that the normal cholesterol level which is viewed as fundamental factor expanding the danger of Ischemic Heart Disease is observed to be higher even in charge bunch than ordinary qualities.[3] This paper completely contributes the uses of PCA in the field of DICOM-based medicinal picture handling. The outcomes and discourses in this paper displayed to demonstrate its efficiency. This look into paper adds to talk about the means for executing PCA in the field of medicinal picture preparing. This field of medicinal picture handling contains extremely expansive number of utilizations.

Biomedical pictures are ruined with clamor which is viewed as the fundamental issue in restorative science. So, there is a need to de-clamor, characterize and separate highlights from the therapeutic pictures for better conclusion. The fundamental objective of this work is to discover different uses of PCA in the extent of restorative picture preparing. PCA definition can be utilized as a computerized picture pressure calculation with a low dimension of misfortune. This examination work watched PCA is utilized for picture combination, picture enrollment, picture division, picture pressure or evacuation of repetitive data, commotion expulsion and highlight extraction. The exhibited outcomes and dialogs demonstrated likewise its effectiveness in different restorative imaging applications. Despite the fact that restorative picture handling is a dynamic field of research and PCA is a standout amongst the most often utilized factual technique in this field, this work will anticipate investigating numerous new uses of PCA in the field of therapeutic picture preparing in not so distant future.

[4] Vital Component Analysis (PCA) of Alzheimer's ailment $(\mathrm{AD})$ and twelve epidemiological and financial parts of the USA states educate etiologic by separating expansive scale designs. The twelve parts exhibit straightforward pair wise Pearson relationships to $\mathrm{AD}$ and after that are dissected by PCA for loadings related with $\mathrm{AD}$. Tedious factor examination and the separating of faulty information diminished the variables (all per capita) related with AD to two, one factor with the segments dental specialists and wine utilization and another factor with the segments brew utilization and dental specialists. Dental specialists and wine are likely connected with decreased $\mathrm{AD}$ rate due to the known backwards relationship with raised instruction. Dental consideration is known to be conversely connected with AD occurrence. The commitment of brew utilization to $\mathrm{AD}$ occurrence is likely a direct result of the negative impact on the inborn invulnerable framework from either phytoestrogen in bounces, or rubbish from aging allowing the parasitic colonization found in $\mathrm{AD}$ minds.

[5] This paper centers on late advancements in AI which have had critical effects in the recognition and finding of different infections. This paper gives a thought of later counterfeit clever frameworks accessible for discovery and analysis of various ailments.

This exploration paper adds to talk about the means for actualizing PCA in the field of therapeutic picture handling. This field of therapeutic picture preparing contains extensive number of uses. Biomedical pictures are undermined with commotion which is viewed as the fundamental issue in therapeutic science. The primary objective of this work is to discover different utilizations of PCA in the extent of therapeutic picture preparing. PCA definition can be utilized as an advanced picture pressure calculation with a low dimension of misfortune [6].

Blue Eyes Intelligence Engineering 
[7] In this paper, Chaman Lal Sabharwal and Bushra Anjum use PCA based calculations in two differing classes, subjective spatial thinking (QSR) to accomplish lossless information decrease and wellbeing informatics to accomplish information decrease alongside improved relapse examination individually. In a versatile cross breed approach, they have utilized PCA to customary relapse calculations to improve their execution and portrayal.Principal segments investigation is normally utilized as one stage in a progression of examinations. They use foremost segments investigation to lessen the quantity of factors and keep away from multi co linearity, or when they have an excessive number of indicators in respect to the quantity of perceptions. They have utilized PCA in two different kinds, QSR and Health Informatics to improve conventional information decrease and calculations. QSR utilizes 9-Intersection model to decide topological relations between spatial articles. All in all, PCA uses numerical information for investigation and as QSR information is intelligent bivalent, they mapped the sensible information to numerical information. This yields over $55 \%$ productivity in execution time. This examination work watched PCA is utilized for picture combination, picture enlistment, picture division, picture pressure or expulsion of repetitive data, commotion evacuation and highlight extraction. The exhibited outcomes and exchanges demonstrated additionally its effectiveness in different medicinal imaging applications.

[8] This paper introduces an overview of the uses of PCA in the field of restorative picture preparing. In this contemplate, different restorative picture application-based PCA results are shown to demonstrate its effectiveness. The PCA gives an incredible asset to information investigation and example acknowledgment, which is frequently utilized in flag and picture preparing. PCA is an established measurable strategy and considered as straight change so it is utilized in information investigation and pressure. PCA is in view of the factual portrayal of an irregular variable. Since, biomedical pictures are ruined with clamor which is viewed as the primary issue in therapeutic science. So there is a need to de-commotion, characterize and separate highlights from the restorative pictures for better analysis. The principle objective of this work is to discover different uses of PCA in the extent of therapeutic picture preparing. The exhibited outcomes indicated likewise its effectiveness in different therapeutic imaging applications. Though restorative picture preparing is an energetic field of research and PCA is one of the most every now and again utilized factual strategy in this field, this work will anticipate investigate numerous new utilizations of PCA in the field of medicinal picture handling in close future.

[9] In this paper, we have assessed a calculation utilizing Principal Component Analysis (PCA) for its application in information examination. In the examination field, it is extremely hard to comprehend the extensive measure of information and is very tedious as well. The technique for PCA is utilized to pack the greatest measure of data into initial two sections of the changed grid known as the key parts by ignoring different vectors that conveys the insignificant data or excess information. They proposed a calculation that is utilized to decrease the component of the

first information did for information examination from 11measurements to 2-dimensional informational index. The objective of the calculation is to restrain the most extreme data just in the initial two segments called as key segments and disregard the remainder of the segments conveying the irrelevant measure of data. To decrease the dimensionality of the information, they have utilized PCA that gives better outcomes.

[10] In this paper, a Computer Aided Diagnosis (CAD) framework is proposed to give a far reaching systematic technique to separating the most noteworthy highlights of Alzheimer's infection (AD). It comprises of three phases: include determination, highlight extraction, and arrangement. This proposition chooses the highlights that have diverse power level at all pictures and disposing of the highlights that have a similar force level to achieve the less subset of highlights that have the most effect unmistakable of AD. At that point decreases the highlights by proposing another element extraction calculation that limits intra independently separation of AD includes. At last, a Linear Support Vector Machine (SVM) classifier was utilized to perform paired arrangements among $\mathrm{AD}$ patients. The outcomes demonstrate that the most noteworthy arrangement execution is acquired utilizing the proposed model and this is promising contrasted with Principle Component Analysis (PCA) and Linear Discriminate Analysis (LDA).In the examinations done by Srimanip.k and kotim.s,the execution investigation of PCA channels and six grouping calculations on the medicinal information (Hepatitis) which happens to be multidimensional and of high measurement with complexities significantly more than the customary information. A valuable computational apparatus for this reason for existing is the present information grouping approach: (I) first we record all the medicinal parameters that describe an infection or a class of ailments, and attempt to characterize them in various bunches equivalent with the quantity of conceivable finding and (ii) knowing the correct determination for each record. Along these lines we discover the rate precision of grouping. At long last, it is reasoned that (I) all the bunching calculations with the exception of Cobweb perform well without PCA channel. (ii) Farthest first is viewed as the best bunching calculation which has minimal estimation of inaccurately grouped examples among others and (iii) PCA channel need not be suggested for medicinal information all in all on account of bunching examination however for some therapeutic information it might turn out to be valuable. At long last, it is reasoned that the consequences of the examination would be successful for the early expectation of the sicknesses so the survival rate could be definitely improved [11].

[12] They quantified the separation between two such populaces utilizing Mahalanobis remove and picked the eigenvectors to expand it, a changed PCA technique, which they call the discriminate PCA (DPCA). DPCA was connected to dissemination tensor based partial anisotropy pictures to recognize age-coordinated schizophrenia subjects 


\section{ADVANCEMENT OF PRINCIPAL COMPONENT JUDGMENT FOR THE CLASSIFICATION AND PREDICTION OF ALZHEIMER'S DISEASE}

from solid controls. The execution of the proposed technique was assessed by the one-forget strategy. They demonstrate that for this partial anisotropy informational index, the characterization blunder with 60 segments was near the base mistake and that the Mahalanobis remove was twice as expansive with DPCA, than with PCA. At last, by concealing the discriminate work with the white issue tracts of the John Hopkins University chart book, they distinguished left predominant longitudinal fasciculus as the tract which gave the least arrangement blunder. What's more, with six ideally picked tracts the arrangement mistake was zero. In this paper we demonstrated that the DPCA technique for requesting eigenvectors is better than the regular PCA requesting, when the basic role of an examination is order of subjects in two gatherings. In the case of the DTI informational index DPCA was extensively superior to PCA when the full informational index was utilized for motivations behind characterization and just possibly better for the forget one technique for assessing the order execution. The proposed technique ought to be considered as an option in contrast to standard PCA while breaking down high dimensionality informational collections it is critical to hold bunch contrasts in the exploratory dimensionality decrease step.

\section{ACKNOWLEDGEMENTS}

"This work has been carried out at DST-FIST sponsored Wireless Sensor Network and IOT Lab (order Sanction o.: SR/FST/ETI-413/2018 Dated: $06^{\text {th }}$ February, 2019), Department of Computer Science and Engineering, Sathyabama Institute of Science and Technology."

\section{REFERENCES}

1. Disease Classification and Prediction using Principal Component Analysis and Ensemble Classification Framework, "Vanishri Arun, Arunkumar B V,Padma S K,ShyamV”, "Research Gate",2017.

2. Application of Principal Component Analysis (PCA) to Medical Data,"Naeem Ahmed Qureshi, Velo Suthar, Habibullah Magsi, Muhammad Javed Sheikh, Mubeena Pathan and Barkatullah Qureshi",Indian Journal of Science and Technology, Vol 10(20), DOI: 10.17485/ijst/2017/v10i20/91294, May 2017.

3. Principal component analysis (pca) in medical image processing using digital imaging and communications in medicine (dicom) medical images,"dr. rm.vidhyavathi,"international journal of pharma and bio sciences", 2017.

4. Principal Component Analysis Applied to Alzheimer's Disease:USA by State," Bodo Parady, Children's Hospital Oakland Research I 1 nstitute, Oakland, CA, USA",2017.

5. A study on Deep Machine Learning Algorithms for diagnosis of diseases,"Dinu A.J, Ganesan, Felix Joseph and Balaji “, International Journal of Applied Engineering Research ISSN 0973-4562 Volume 12, Number 17 (2017) pp. 6338-6346.

6. Dr. RM.vidhyavathi," Principal Component Analysis (PCA) in medical image processing using digital imaging and communications in medicine (dicom) medical images", International Journal of Pharma and Bio Sciences,2017.

7. Data Reduction and Regression Using Principal Component Analysis in Qualitative Spatial Reasoning and Health Informatics,"Chaman Lal Sabharwal and Bushra Anjum", 2016.
8. Principal component analysis in medical image processing: a study,"Dibyadeep Nandi, Amira S. Ashour*, Sourav Samanta, Sayan Chakraborty, Mohammed A.M. Salem, Nilanjan Dey", Int. J. Image Mining, Vol. 1, No. 1, 2015.

9. Data Analysis Using Principal Component Analysis,"ShrutiSehgal,Harpreet Singh, Mohit Agarwal V. Bhasker Shantanu",2014 International Conference on Medical Imaging, m-Health and Emerging Communication Systems (MedCom).

10. Selecting and Extracting Effective Features for Automated Diagnosis of Alzheimer's Disease, "Mohamed M. Dessouky,Mohamed A. Elrashidy, Taha E. Taha,Hatem M. Abdelkader", International Journal of Computer Applications (0975 - 8887) Volume 81 - No.4, November 2013.

11. Srimanip.k,kotim.s.," Evaluation of Principal Components Analysis (PCA) and Data Clustering Techniques (DCT) on medical data", International journal of knowledge engineering, 2012 .

12. Application of Principal Component Analysis to Distinguish Patients with Schizophrenia from Healthy Controls Based on Fractional Anisotropy Measurements, "A. Caprihan, G.D. Pearlson and V.D. Calhoun","Elseiver",2008.

13. Alzheimer's Association |Alzheimer's disease and Dementia, 2015. 〈http://www. Alz.org/〉 (Accessed 5 April 2015).

14. A. Association, Alzheimer' s Association Report 2015 Alzheimer' s disease facts and figures, Alzheimer's. Dement. 11 (2015) 332-384. http://dx.doi.org/10.1016/ j.jalz.2015.02.003

\section{AUTHORS PROFILE}

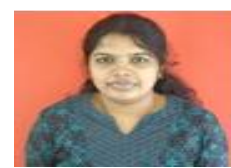

First Author: M.S.Roobini, Department of Computer Science and Engineering, Sathyabama Institute of Science and Technology, Chennai, India, roobinims@gmail,com

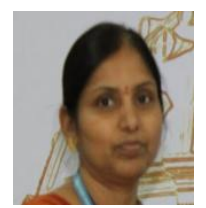

Second Author: Dr.M.Lakshmi, Principal, SriKrishna College of Technology, Coimbatore, Chennai, India

laks@icadsindia.com 